\title{
Electrochemical Properties of Seamless Three-Dimensional Carbon Nanotubes-Grown Graphene Modified with Horseradish Peroxidase
}

Kikuo Komori, ${ }^{1,2 *}$ Trupti Terse-Thakoor, ${ }^{3}$ and Ashok Mulchandani ${ }^{2 *}$

1. Institute of Industrial Science, University of Tokyo, Komaba, Meguro-ku, Tokyo 153-8505, Japan.

2. Department of Chemical and Environmental Engineering, University of California, Riverside, California 92521, United States.

3. Department of Bioengineering, University of California, Riverside, California 92521, United States.

Corresponding author:

Ashok Mulchandani, adani@engr.ucr.edu

Kikuo Komori, kkomori@iis.u-tokyo.ac.jp

\begin{abstract}
Horseradish peroxidase (HRP) was immobilized through sodium dodecyl sulfate (SDS) on the surface of a seamless three-dimensional hybrid of carbon nanotubes grown at the graphene surface (HRP-SDS/CNTs/G) and its electrochemical properties were investigated. Compared with graphene alone electrode modified with HRP via SDS (HRP-SDS/G electrode), the surface coverage of electroactive HRP at the CNTs/G electrode surface was approximately 2-fold greater because of CNTs grown at the graphene surface. Based on the increase in the surface coverage of electroactive HRP, the sensitivity to $\mathrm{H}_{2} \mathrm{O}_{2}$ at the HRP-SDS/CNTs/G electrode was higher than that at the HRP-SDS/G electrode. The kinetics of the direct electron transfer from the CNTs/G electrode to compound I and II of modified HRP was also analyzed.
\end{abstract}

KEYWORDS: carbon nanotubes, graphene, hybrid, horseradish peroxidase, biosensing

\section{INTRODUCTION}

Seamless three-dimensional (3D) $\mathrm{sp}^{2}$ carbon-based nanomaterials, such as foam-like graphene $[1,2]$ and carbon nanotubes-grown graphene $(\mathrm{CNTs} / \mathrm{G})$ hybrid 
materials [3, 4], have recently attracted great attention not only because of their high electrical conductivity but also their high surface area per unit planar/footprint area. Additionally, since charge carriers are movable in all three dimensions without significant contact resistance, such seamless 3D $\mathrm{sp}^{2}$ carbon-based nanomaterials are expected to provide electrode materials for high power fuel cells as well as highly sensitive sensors, compared with seamless $2 \mathrm{D} \mathrm{sp}{ }^{2}$ carbon-based nanomaterials, such as graphene. Also, because of the flat surface of its backside, the CNTs/G hybrid has the potential for the development of flexible and wearable biosensors for physiological monitoring.

Redox enzymes, such as horseradish peroxidase (HRP) and glucose oxidase (GOx), are widely used for electrochemical biosensors. However, they are generally difficult to directly communicate with flat metal electrodes due to the deeply embedded active site in the thick insulating polypeptide layer. Meanwhile, it is known that CNTs and graphene flakes can communicate electrochemically with redox enzymes directly, because of their small nanostructure with high conductivity [5-11]. Such carbon nanomaterials have therefore been expected to be utilized for the development of enzyme-based electrochemical biosensors. However, properties of CNTs and graphene emerge only in one direction for CNTs and planar direction for graphene due to their one- and two-dimensional structures, respectively [12-14]. Therefore, controlling the orientation of those carbon nanomaterials is necessary. Additionally, there are also some non-trivial problems, such as the aggregate formation and significant contact resistance among CNTs and graphene flakes.

Toward the development of highly sensitive enzyme-based electrochemical biosensors without the above problems, we previously constructed CNTs/G hybrid electrodes modified with GOx [15] or heme peptide (HP) [16], which has a similar structure to the active center of peroxidase and has often been used for $\mathrm{H}_{2} \mathrm{O}_{2}$ sensing [17-19]. However, the catalytic activity of HP is lower than that of HRP and therefore HRP-based biosensor would be more sensitive compared to one based on HP. Thus, is an interest in using the full $\mathrm{HRP}$ for $\mathrm{H}_{2} \mathrm{O}_{2}$ sensing. In the present work, we HRP-modified CNTs/G electrodes and studied the kinetics of electron transfer from the $\mathrm{CNTs} / \mathrm{G}$ hybrid to peroxidase. Based on the previous report by Yan et al., we used surfactant sodium dodecyl sulfate (SDS) to immobilize HRP at the CNTs/G [6]. Furthermore, based on the direct reduction of $\mathrm{HRP}(\mathrm{P}) \mathrm{Fe}^{4+}$-oxygen complexes ( $\mathrm{P}$ : 
porphyrin ring), such as compound I and II, in the presence of $\mathrm{H}_{2} \mathrm{O}_{2}$ at sufficiently higher potential relative to the formal potential of the HRP(P)Fe ${ }^{2+/ 3+}$ couple $[20,21]$, we compared catalytic reduction currents of $\mathrm{H}_{2} \mathrm{O}_{2}$ at the HRP-modified CNTs/G electrode with those at HRP-modified graphene electrode (Figure 1). Thus, we confirmed the superiority of CNTs/G hybrid films in electrochemical biosensing, compared with graphene films.

\section{"Here Fig. 1"}

\section{EXPERIMENTAL}

\subsection{Preparation of $C N T s / G$ Films}

$\mathrm{CNTs} / \mathrm{G}$ hybrid was prepared according to the previous report [16]. Briefly, a copper $(\mathrm{Cu})$ foil $\left(\sim 6.5 \mathrm{~cm}^{2}\right.$ and $25 \mu \mathrm{m}$ thick), which is a catalyst and substrate for graphene growth, was placed inside a quartz tube (ca. $3 \mathrm{~cm}$ in inner diameter and ca. 75 cm long) and annealed at $1030{ }^{\circ} \mathrm{C}$ under flowing $\mathrm{Ar}(190 \mathrm{sccm})$ and $\mathrm{H}_{2}(10 \mathrm{sccm})$ at atmospheric pressure in an electric furnace (Lindberg/Blue $\mathrm{M}^{\mathrm{TM}}$ Mini-Mite ${ }^{\mathrm{TM}}$ Tube Furnace, Thermo Scientific). At this time $\mathrm{CH}_{4}(5 \mathrm{sccm})$ was introduced in the tube for 9 min to grow graphene followed by cooling of the furnace to room temperature in flowing $\mathrm{Ar} / \mathrm{H}_{2}$ atmosphere. Subsequently, the graphene-formed $\mathrm{Cu}$ foil was decorated with $1.5 \mathrm{~nm}$ thick iron film, which acted as catalysts for CNTs growth, using an e-beam evaporator (Temescal BJD-1800, Technical Engineering Services). The graphene/Cu foil with the iron film was placed back in the quartz tube and heated to $750{ }^{\circ} \mathrm{C}$ in flowing $\operatorname{Ar}(100 \mathrm{sccm})$ and $\mathrm{H}_{2}(50 \mathrm{sccm})$ atmosphere. After the temperature was stabilized at $750{ }^{\circ} \mathrm{C}$, a $15 \mathrm{sccm}$ flow of $\mathrm{C}_{2} \mathrm{H}_{2}$ was supplied in the tube for $5 \mathrm{~min}$, followed by cooling of the furnace to room temperature in flowing $\mathrm{Ar} / \mathrm{H}_{2}$ atmosphere. The graphene film formed on the backside of $\mathrm{CNTs} / \mathrm{G} / \mathrm{Cu}$ foil was removed by $\mathrm{O}_{2}$ plasma, followed by etching of the $\mathrm{Cu}$ foil in a $1 \mathrm{M} \mathrm{FeCl}_{3}$ aqueous solution to obtain the CNTs/G hybrid. The CNTs/G hybrid was cleaned with $5 \% \mathrm{HCl}$ solution to completely remove the $\mathrm{Cu}$ foil and the iron nanoparticles. Note that we also obtained the graphene film using the same procedure. Fundamental properties of $\mathrm{CNTs} / \mathrm{G}$ and graphene films were reported previously $[15,16]$.

\subsection{Preparation of HRP-Modified Electrodes}


A glassy carbon (GC) electrode (3 $\mathrm{mm}$ in diameter, ALS Co., Ltd) was polished with 1 and $0.05 \mu \mathrm{m}$ alumina slurries on a polishing cloth and then thoroughly rinsed with distilled water, followed by sonication in 2-propanol and distilled water, respectively. After drying with a high-purity nitrogen stream, the CNTs/G or graphene was transferred to the GC electrode surface and then kept at $50{ }^{\circ} \mathrm{C}$ in an electric oven for $1 \mathrm{~h}$ to be firmly attached on the GC surface. Subsequently, the CNTs/G and graphene electrodes were immersed in either $\mathrm{A}) \mathrm{pH}$ 7, $67 \mathrm{mM}$ phosphate buffer (PB) containing $0.5 \mathrm{mM}$ HRP (Sigma-Aldrich) with $20 \mathrm{mM} \mathrm{SDS}$ for $12 \mathrm{~h}$ at $4{ }^{\circ} \mathrm{C}$ to obtain

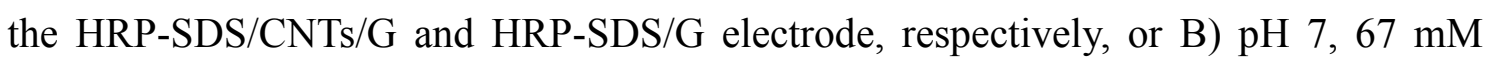
phosphate buffer (PB) containing $0.5 \mathrm{mM}$ HRP (Sigma-Aldrich) for $12 \mathrm{~h}$ at $4{ }^{\circ} \mathrm{C}$ to obtain the HRP/CNTs/G and HRP/G electrode, respectively.

\subsection{Electrochemical Measurements}

Electrochemical measurements were performed with a potentiostat Versa STAT (Princeton Applied Research, USA) in $67 \mathrm{mM}$ phosphate buffer (pH 7.4) in a batch system. $\mathrm{A} \mathrm{Ag}|\mathrm{AgCl}| \mathrm{KCl}$ (sat.) and a coiled platinum wire were used as reference and counter electrodes, respectively. The catalytic activity of HRP-SDS/CNTs/G and HRP-SDS/G electrodes toward $\mathrm{H}_{2} \mathrm{O}_{2}$ reduction was evaluated by amperometric measurements. After the working electrode was polarized at $+150 \mathrm{mV}$ and a steady-state current was obtained, a $\mathrm{H}_{2} \mathrm{O}_{2}$ solution was added into the electrolyte solution. From the steady-state current obtained here, the catalytic activity of HRP at the CNTs/G and graphene electrodes was determined.

\section{RESULTS AND DISCUSSION}

\subsection{Electrochemical Characterization}

Cyclic voltammetry (CV) were performed at the $\mathrm{G}, \mathrm{CNT} / \mathrm{G}, \mathrm{HRP} / \mathrm{G}$, HRP/CNTs/G, HRP-SDS/G and HRP-SDS/CNTs/G electrodes in deaerated PB. As shown in Figure 2A, there was no apparent background redox peak at both the CNT/G and graphene electrodes. Similarly, the HRP/CNTs/G and HRP/G electrodes also did not show redox peak (data not shown). On the other hand, redox peaks appeared at both the HRP-SDS/G and HRP-SDS/CNTs/G at approximately $-320 \mathrm{mV}$ (vs. $\mathrm{Ag} \mid \mathrm{AgCl}$ ), corresponding to $(\mathrm{P}) \mathrm{Fe}^{2+/ 3+}$. These findings are similar to reported by Yan et al. [6] and as in their case can be ascribed to properties of the SDS for facilitating protein 
electrochemistry and the structural properties of CNT and the 3D morphology of the $\mathrm{CNT} / \mathrm{G}$ electrode. We also evaluated cetyltrimethylammonium bromide (CTAB) for the immobilization of HRP in the phosphate buffer ( $\mathrm{pH}$ 7.4). However, no redox peaks of (P)Fe $\mathrm{Fe}^{2+/ 3+}$ were clearly observed. Since CTAB is a cationic surfactant and the isoelectric point of HRP is 8.9 , HRP might not be immobilized through CTAB probably due to the electrostatic repulsion between CTAB and HRP. We therefore used only SDS modification for HRP immobilization for further studies.

The redox peak current $\left(j_{\mathrm{p}}\right)$ for both electrodes was proportional to the scan rate $(v)$ below at least $1.5 \mathrm{~V} \mathrm{~s}^{-1}$ (Figure 2B), indicating that the redox reaction was a surface controlled process. In addition, the redox peak current at the HRP-SDS/CNTs/G electrode was approximately 2-times larger than that at the HRP-SDS/G electrode. This is in accordance with the previously reported $\sim 2.5$-fold larger apparent electroactive surface area for the $\mathrm{CNTs} / \mathrm{G}$ film compared to graphene because of CNTs grown at the graphene surface [16]. Since the peak current was proportional to the scan rate below at least $1.5 \mathrm{~V} \mathrm{~s}^{-1}$ (Figure 2B), the surface coverage $\Gamma$ of electroactive HRP at both CNTs/G and graphene electrodes was determined from the equation,

$$
I_{\mathrm{P}}=n^{2} F^{2} \Gamma \mathcal{v} / 4 R T
$$

where $n$ is the number of electrons transferred $(n=1), F$ is the Faraday constant, $v$ is the scan rate, $R$ is the gas constant, and $T$ is the temperature. Based on equation $1, \Gamma$ was estimated to be $\sim 4.3 \times 10^{-11} \mathrm{~mol} \mathrm{~cm}^{-2}$ for the HRP-SDS/CNTs/G electrode and $2.1 \times$ $10^{-11} \mathrm{~mol} \mathrm{~cm}^{-2}$ for the HRP-SDS/G electrode. On the basis of the reported $\Gamma$ value of $5 \times$ $10^{-11} \mathrm{~mol} \mathrm{~cm}^{-2}$ for the HRP monolayer-modified basal plane pyrolytic graphite (BPPG) electrode through didodecyldimethylammonum bromide [22], we hypothesize that HRP molecules formed a monolayer at the graphene and CNTs/G surfaces.

\section{"Here Fig. 2"}

As mentioned above, the present redox reaction of HRP is a surface controlled process. In addition, as the scan rate increased, the peak-to-peak separation $\left(\Delta E_{\mathrm{p}}\right)$ also gradually increased. To obtain the kinetic parameter for HRP redox, the apparent heterogeneous electron transfer rate constant $k_{\text {app }}$ was therefore calculated according to the Laviron's theory [23]. The anodic and cathodic peak potential $E_{\mathrm{pa}}$ and $E_{\mathrm{pc}}$ can be 
represented as follows:

$$
\begin{aligned}
& E_{\mathrm{pa}}=E^{0}-(2.3 R T / \alpha n F) \log (\alpha / m) \\
& E_{\mathrm{pc}}=E^{0}+\{2.3 R T /(1-\alpha) n F\} \log \{(1-\alpha) / m\} \\
& m=R T k_{\text {app }} / F n v
\end{aligned}
$$

where $\alpha$ is the transfer coefficient. Based on the plots of $E_{\mathrm{pa}}$ or $E_{\mathrm{pc}}-E_{0}$ versus $\log v, \alpha$ can be determined from the slopes of the anodic and cathodic processes (Figure 3 ) to be about $0.50 \pm 0.02$ and $0.41 \pm 0.04$ for HRP-SDS/CNTs/G and HRP-SDS/G, respectively. According to the equations $2-4, \Delta E_{\mathrm{p}}$ can be obtained by the following equation, which is accepted above $200 \mathrm{mV}$ :

$$
\begin{gathered}
\Delta E_{\mathrm{p}}=\{2.3 R T /(1-\alpha) \alpha n F\}\left\{\alpha \log (1-\alpha)+(1-\alpha) \log \alpha-\log (R T / n F)-\log k_{\text {app }}\right\}+\{2.3 R T /(1 \\
-\alpha) n F\} \log v
\end{gathered}
$$

Based on this equation, $k_{\text {app }}$ was determined to be $19.2 \pm 2.5 \mathrm{~s}^{-1}$ for the HRP-SDS/CNTs/G electrode and $15.7 \pm 2.3 \mathrm{~s}^{-1}$ for the HRP-SDS/G electrode, respectively. The difference in $k_{\text {app }}$ can be ascribed to a small amount of defects and/or graphene edge sites in the $\mathrm{CNTs} / \mathrm{G}$ hybrid. It is known that heterogeneous electron transfer reactions of redox species at edge-oriented pyrolytic graphite (EOPG) electrodes are generally faster than those at BPPG electrodes [24-27]. In addition, it is known that the heterogeneous electron transfer reaction rate is accelerated at carbon nanofibers with stacked graphene, which provided a high density of the edge site at their surfaces $[28,29]$. As reported previously, the existence of defects and/or graphene edge sites in the CNTs/G hybrid were confirmed from Raman spectroscopy, whereas the graphene film formed a monolayer sheet with very few defects and edge sites [16]. Thus, the heterogeneous electron transfer rate of HRP might be facilitated at the $\mathrm{CNTs} / \mathrm{G}$ in comparison with the graphene. Meanwhile, the $k_{\text {app }}$ value obtained here was larger than that for previously reported HRP-SDS/CNTs $\left(3.5 \mathrm{~s}^{-1}\right)$ [6] and HRP-Nafion/G $\left(4.63 \mathrm{~s}^{-1}\right)$ [30] electrodes. The present $\mathrm{CNTs} / \mathrm{G}$ and graphene electrodes were basically the seamless structure, whereas the previous CNTs and graphene electrodes consisted of their nanotubes and nanoflakes assembled on the GC electrode. Compared with the assembled structure, the seamless structure might facilitate the electron transfer to some extent.

\section{"Here Fig. 3"}




\subsection{Catalytic Reduction of $\mathrm{H}_{2} \mathrm{O}_{2}$}

Figure 4 shows typical cathodic current responses $(\Delta j)$ to $\mathrm{H}_{2} \mathrm{O}_{2}$ at CNTs/G and graphene electrodes, on which HRP was immobilized via SDS. The cathodic current at the HRP-SDS/CNTs/G and HRP-SDS/G electrodes was significantly larger than that at $\mathrm{CNTs} / \mathrm{G}$ and graphene electrodes without HRP. This considerably enhanced current responses at enzyme modified electrodes can be credited to electron transfer from $\mathrm{CNTs} / \mathrm{G}$ and graphene electrodes to HRP. The possible reaction mechanisms are proposed as follows [20, 21].

$$
\begin{aligned}
& \text { Ferric-HRP }+\mathrm{H}_{2} \mathrm{O}_{2} \rightarrow \text { Compound } \mathrm{I}+\mathrm{H}_{2} \mathrm{O} \\
& \text { Compound } \mathrm{I}+\mathrm{e}^{-}+\mathrm{H}^{+} \rightarrow \text { Compound II } \\
& \text { Compound } \mathrm{II}+\mathrm{e}^{-}+\mathrm{H}^{+} \rightarrow \text { Ferric-HRP }+\mathrm{H}_{2} \mathrm{O}
\end{aligned}
$$

where compound I and II are oxidized complexes of HRP, such as $\left[(\mathrm{HRP}) \mathrm{Fe}^{4+}=\mathrm{O}\right]^{+\bullet}$ and $\left[(\mathrm{HRP}) \mathrm{Fe}^{4+}-\mathrm{OH}\right]^{+}$, respectively. Further, as shown in Fig. 4, the cathodic current at the HRP-SDS/CNTs/G and HRP-SDS/G electrodes increased and then leveled off, as the concentration of $\mathrm{H}_{2} \mathrm{O}_{2}$ increased. The initial linear increase of current as a function of $\mathrm{H}_{2} \mathrm{O}_{2}$ concentration suggests the reaction 6 to be rate determining. On the other hand, the subsequent plateauing of current points to reaction 7 and/or 8 to be rate determining. It is to be noted, here the current response was not influenced when the electrolyte solution was stirred, an indication the catalytic cathodic currents obtained at both HRP-SDS/CNTs/G and HRP-SDS/G electrodes were kinetically controlled. Therefore, all electroactive HRP molecules should contribute to the current. The cathodic current response at the HRP-SDS/CNTs/G electrode was about 2-fold larger than that at the HRP-SDS/G electrode, reflecting that the surface coverage of electroactive HRP at the former is about 2 times larger than that at the latter, as mentioned above.

\section{"Here Fig. 4"}

\subsection{Analysis of Reaction Kinetics}

Based on the above findings that cathodic current was not diffusion controlled and was proportional to the surface coverage of electrocative HRP molecules, the rate constants for the three reactions (equations 6 to 8 ) can be calculated using the equation $9[20,21,31]$,

$$
i=2 F \Gamma /\left[1 / k_{1} C_{\mathrm{S}}+\left(k_{2}+k_{3}\right) / k_{2} k_{3} C_{\mathrm{H}}\right]
$$


where $k_{1}, k_{2}$, and $k_{3}$ are the rate constant of reactions $6-8, C_{\mathrm{S}}$ and $C_{\mathrm{H}}$ are the $\mathrm{H}_{2} \mathrm{O}_{2}$ and proton concentration in the bulk solution, respectively. At low $\mathrm{H}_{2} \mathrm{O}_{2}$ concentrations (linear range of current vs. $\mathrm{H}_{2} \mathrm{O}_{2}$ concentration plot in Fig. 4), when almost all HRP is in ferric state, the current response is determined by the rate of reaction 6 and therefore given by the equation $[20,21]$

$$
i_{1}=2 F \Gamma k_{1} C_{\mathrm{S}}
$$

Based on this equation, the rate constant $k_{1}$ for HRP-SDS/CNTs/G and HRP-SDS/G electrodes were estimated to be approximately 370 and $150 \mathrm{M}^{-1} \mathrm{~s}^{-1}$, respectively. Based on the fact that HRP was immobilized through SDS at essentially the same geometrical structure of $\mathrm{sp}^{2}$ bonded carbon, we predicted the $k_{1}$ value at the HRP-SDS/CNTs/G and HRP-SDS/G electrodes to be almost the same. The reason for a larger $k_{1}$ at the HRP-SDS/CNTs/G is unclear. A plausible explanation could be an enhanced conformational stability of HRP on the nanofiber structure of CNTs grown at the graphene compared with the flat graphene. Meanwhile, $k_{1}$ for the HRP-SDS/CNTs/G electrode was larger than that for CNTs/G electrode modified with HP (ca. $120 \mathrm{M}^{-1} \mathrm{~s}^{-1}$ ) reported previously [16] because of the difference in molecular complexity. However, the $k_{1}$ values obtained here were much smaller than the value for dissolved HRP $(1.8 \times$ $10^{7} \mathrm{M}^{-1} \mathrm{~s}^{-1}$ ) [32]. A reason is the likely denaturation of HRP at CNTs/G and graphene due to its strong adsorption via SDS.

At higher $\mathrm{H}_{2} \mathrm{O}_{2}$ concentrations (plateau region of current vs. $\mathrm{H}_{2} \mathrm{O}_{2}$ concentration plot in Fig. 4), assuming that all the HRP molecules are existing as compound I or II and the reaction is not controlled by proton diffusion but the enzymatic reaction, the cathodic current $i_{23}$ is given by the equation [20,21]

$$
i_{23}=2 F \Gamma k_{2} k_{3} C_{\mathrm{H}} /\left(k_{2}+k_{3}\right)
$$

Based on the equation 11 , the $k_{2} k_{3} /\left(k_{2}+k_{3}\right)$ value at the HRP-SDS/CNTs/G electrode was calculated to be about $7.6 \times 10^{5} \mathrm{M}^{-1} \mathrm{~s}^{-1}$ which was slightly larger than that at the HRP-SDS/G electrode (ca. $6.2 \times 10^{5} \mathrm{M}^{-1} \mathrm{~s}^{-1}$ ). In addition, the values of the apparent heterogeneous rate constant, $C_{\mathrm{H}} k_{2} k_{3} /\left(k_{2}+k_{3}\right)$, at $\mathrm{pH} 7.4$ were estimated to be $\sim 3.0 \times$ $10^{-2}$ and $2.5 \times 10^{-2} \mathrm{~s}^{-1}$ for HRP-SDS/CNTs/G and HRP-SDS/G electrodes, respectively. This difference is likely due to the existence of the small amount of defects and/or edge sites in the $\mathrm{CNTs} / \mathrm{G}$, as mentioned above. Actually, the $k_{2} k_{3} /\left(k_{2}+k_{3}\right)$ values obtained here were one order of magnitude smaller than that obtained at $\mathrm{pH} 7.0$ for the HRP-adsorbed pyrolytic graphite electrode reported by Razgus et al. $\left(6.6 \times 10^{6} \mathrm{M}^{-1} \mathrm{~s}^{-1}\right)$ 
[33]. In contrast, the obtained values were larger than that at a HRP-adsorbed graphite powder-coated electrode $\left(5.6 \times 10^{2} \mathrm{M}^{-1} \mathrm{~s}^{-1}\right)$ [20]. For the graphite powder coating, a binder is often required for the fabrication of such graphite electrodes. It is known that the binder sometime interferes with the direct electron transfer between enzymes and graphite powder [20]. In the present work, no binder was required for the preparation of CNTs/G modified electrodes. Thus, the direct electron transfer from HRP to the CNTs/G might be accelerated, though the edge sites of graphene were virtually absent. However, the $k_{1}$ value for $\mathrm{CNTs} / \mathrm{G}$ was significantly small, as mentioned above. Due to this, the lower detection limit of $\mathrm{H}_{2} \mathrm{O}_{2}$ for the HRP-SDS/CNTs/G electrode $\left(\sim 10^{-5} \mathrm{M}\right)$ was five orders of magnitude larger than that for the HRP-adsorbed graphite powder-coated electrode $\left(\sim 2 \times 10^{-10} \mathrm{M}\right)$ [20]. By improving this parameter, we strongly believe that $\mathrm{CNTs} / \mathrm{G}$ could be applied for the development of a highly sensitive electrochemical $\mathrm{H}_{2} \mathrm{O}_{2}$ biosensor. For example, the surface modification of the CNTs/G with metal nanoparticles [34, 35] and metal nanosheets [36] might help retain enzymatic activity.

\section{CONCLUSION}

The electrochemical properties of the CNTs/G electrode modified with HRP through SDS were investigated to explore the enhancement of the sensitivity to analyte $\mathrm{H}_{2} \mathrm{O}_{2}$ because of the increase in the surface coverage of electroactive HRP at the CNTs-grown on graphene surface. Compared with the HRP/G electrode, the catalytic current for $\mathrm{H}_{2} \mathrm{O}_{2}$ reduction at the HRP/CNTs/G electrode increased by a factor of about 2. The surface coverage of HRP was about $\sim 4.3 \times 10^{-11} \mathrm{~mol} \mathrm{~cm}^{-2}$ at the CNTs/G electrode. The rate constant $k_{1}$ was estimated to be $\sim 370 \mathrm{M}^{-1} \mathrm{~s}^{-1}$. The overall rate constant of electron transfer reaction $k_{2} k_{3} /\left(k_{2}+k_{3}\right)$ and the apparent heterogeneous rate constant $C_{\mathrm{H}} k_{2} k_{3} /\left(k_{2}+k_{3}\right)$ at $\mathrm{pH} 7.4$ were $\sim 7.6 \times 10^{5} \mathrm{M}^{-1} \mathrm{~s}^{-1}$ and $3.0 \times 10^{-2} \mathrm{~s}^{-1}$, respectively. Thus, we hypothesize that the CNTs/G hybrid would be a good candidate as the conductive nanoscaffold for enzymes toward the development of highly sensitive electrochemical biosensors.

\section{ACKNOWLEDGEMENTS}

KK acknowledges the financial support of Young Researchers Overseas Study

Program for Mechanical System Innovation of University of Tokyo, Japan and Grant-in-Aid for Young Scientist (B) (No. 26790011) from the Ministry of Education, 
Culture, Sports, Science, and Technology (MEXT). AM acknowledges the financial support of W. Ruel Johnson Chair in Environmental Engineering, USDA (2014-67021-21589) and NSF (1307671).

\section{REFERENCES}

1. Z. Chen, W. Ren, L. Gao, B. Liu, S. Pei, and H.-M. Cheng, Three-dimensional flexible and conductive interconnected graphene networks grown by chemical vapor deposition, Nat. Mater. 10 (2011) 424-428.

2. X. Wang, Y. Zhang, C. Zhi, X. Wang, Z. Tang, Y. Xu, Q. Weng, X. Jiang, M. Mitome, D. Golberg, and Y. Bando, Three-dimensional strutted graphene grown by substrate-free sugar blowing for high-power-density supercapacitors, Nat. Commun. 4 (2013) 2905.

3. R. K. Paul, M. Ghazinejad, M. Penchev, J. Lin, M. Ozkan, C. S. Ozkan, Synthesis of a pillared graphene nanostructure: a counterpart of three-dimensional carbon architectures, Small 6 (2010) 2309-2313.

4. Y. Zhu, L. Li, C. Zhang, G. Casillas, Z. Sun, Z. Yan, G. Ruan, Z. Peng, A.-R. O. Raji, C. Kittrell, R. H. Hauge, and J. M. Tour, A seamless three-dimensional carbon nanotube graphene hybrid material, Nat. Commun. 3 (2012) 1225.

5. G.-C. Zhao, L. Zhang, X.-W. Wei, and Z.-S. Yang, Myoglobin on multi-walled carbon nanotubes modified electrode: direct electrochemistry and electrocatalysis, Electrochem. Commun. 5 (2003) 825-829.

6. Y. Yan, W. Zheng, M. Zhang, L. Wang, L. Su, and L. Mao, Bioelectrochemically functional nanohybrids through co-assembling of proteins and surfactants onto carbon nanotubes: facilitated electron transfer of assembled proteins with enhanced faradic response, Langmuir 21 (2005) 6560-6566.

7. X. Zuo, S. He, D. Li, C. Peng, Q. Huang, S. Song, and C. Fan, Graphene oxide-facilitated electron transfer of metalloproteins at electrode surfaces, Langmuir 26 (2010) 1936-1939.

8. Q. Zhang, Y. Qiao, F. Hao, L. Zhang, S. Wu, Y. Li, J. Li, and X.-M. Song, Fabrication of a biocompatible and conductive platform based on a single stranded DNA/graphene nanocomposite for direct electrochemistry and electrocatalysis, Chem. Eur. J. 16 (2010) 8133-8139.

9. M. Tominaga, H. Yamaguchi, S. Sakamoto, and I. Taniguchi, Effect of surface 
oxidized structure of single-walled carbon nanotubes on heterogeneous electron-transfer reaction of cytochrome $c$, Chem. Lett. 39 (2010) 976-977.

10. A. K. M. Kafi and M. J. Crossley, Synthesis of a conductive network of crosslinked carbon nanotube/hemoglobin on a thiol-modified Au surface and its application to biosensing, Biosens. Bioelectron. 42 (2013) 273-279.

11. D. Chen, H. Feng, and J. Li, Graphene oxide: Preparation, functionalization, and electrochemical applications, Chem. Rev. 112 (2012) 6027-6053.

12. F. Patolsky, Y. Weizmann, and I. Willner, Long-range electrical contacting of redox enzymes by SWCNT connectors, Angew. Chem. Int. Ed. 43 (2004) 2113-2117.

13. J. Liu, A. Chou, W. Rahmat, M. N. Paddon-Row, and J. J. Gooding, Achieving direct electrical connection to glucose oxidase using aligned single walled carbon nanotube arrays, Electroanalysis 17 (2005) 38-46.

14. A. Ueda, D. Kato, R. Kurita, T. Kamata, H. Inokuchi, S. Umemura, S. Hirono, and O. Niwa, Efficient direct electron transfer with enzyme on a nanostructured carbon film fabricated with a maskless top-down UV/ozone process, J. Am. Chem. Soc. 133 (2011) 4840-4846.

15. T. Terse-Thakoor, K. Komori, P. Ramnani, I. Lee, and A. Mulchandani, Electrochemically functionalized seamless three-dimensional graphene-carbon nanotube hybrid for direct electron transfer of glucose oxidase and bioelectrocatalysis, Langmuir 31 (2015) 13054-13061.

16. K. Komori, T. Terse-Thakoor, and A. Mulchandani, Bioelectrochemistry of heme peptide at seamless three-dimensional carbon nanotubes/graphene hybrid films for highly sensitive electrochemical biosensing, ACS Appl. Mater. Interfaces 7 (2015) 3647-3654.

17. T. Tatsuma and T. Watanabe, Peroxidase model electrodes: heme peptide modified electrodes as reagentless sensors for hydrogen peroxide, Anal. Chem. 63 (1991) 1580-1585.

18. K. Komori, K. Takada, and T. Tatsuma, Peroxidase model electrodes: self-mediation of heme peptide multilayer-modified electrodes and application to biosensing with adjustable dynamic range, J. Electroanal. Chem. 585 (2005) 89-96.

19. K. Komori, K. Takada, and T. Tatsuma, Electrodes modified with the phase transition polymer and heme peptide: biocatalysis and biosensing with tunable activity and dynamic range, Langmuir 22 (2006) 478-483. 
20. T. Tatsuma, K. Ariyama, and N. Oyama, Kinetic analysis of electron transfer from a graphite coating to horseradish peroxidase, J. Electroanal. Chem. 446 (1998) 205-209.

21. S. Ko, Y. Takahashi, H. Fujita, T. Tatsuma, A. Sakoda, and K. Komori, Peroxidase-modified cup-stacked carbon nanofiber networks for electrochemical biosensing with adjustable dynamic range, RSC Adv. 2 (2012) 1444-1449.

22. X. Chen, X. Peng, J. Kong, and J. Deng, Facilitated electron transfer from an electrode to horseradish peroxidase in a biomembrane-like surfactant film, J. Electroanal. Chem. 480 (2000) 26-33.

23. E. Laviron, General expression of the linear potential sweep voltammogram in the case of diffusionless electrochemical systems, J. Electroanal. Chem. 101 (1979) 19-28.

24. R. M. Wightman, M. R. Deakin, P. M. Kovach, W. G. Kuhrand, and K. J. Stutts, Methods to improve electrochemical reversibility at carbon electrodes, J. Electrochem. Soc. 131 (1984) 1578-1583.

25. R. J. Bowling, R. T. Packard and R. L. McCreery, Activation of highly ordered pyrolytic graphite for heterogeneous electron transfer: relationship between electrochemical performance and carbon microstructure, J. Am. Chem. Soc. 111 (1989) 1217-1223.

26. C. E. Banks, R. R. Moore, T. J. Davies and R. G. Compton, Investigation of modified basal plane pyrolytic graphite electrodes: definitive evidence for the electrocatalytic properties of the ends of carbon nanotubes, Chem. Commun. (2004) 1804-1805.

27. M. Pacios, M. del Valle, J. Bartroli and M. J. Esplandiu, Electrochemical behavior of rigid carbon nanotube composite electrodes, J. Electroanal. Chem. 619-620 (2008) 117-124.

28. A. Ambrosi, A. Bonanni, and M. Pumera, Electrochemistry of folded graphene edges, Nanoscale 3 (2011) 2256-2260.

29. S. Ko, T. Tatsuma, A. Sakoda, Y. Sakai, and K. Komori, Electrochemical properties of oxygenated cup-stacked carbon nanofiber-modified electrodes, Phys. Chem. Chem. Phys. 16 (2014) 12209-12213.

30. M. Li, S. Xu, M. Tan, L. Liu, F. Gao, and Y. Wang, Direct electrochemistry of horseradish peroxidase on graphene-modified electrode for electrocatalytic 
reduction towards $\mathrm{H}_{2} \mathrm{O}_{2}$, Electrochim. Acta 56 (2011) 1144-1149.

31. R. A. Kamin and G. S. Wilson, Rotating ring-disk enzyme electrode for biocatalysis kinetic studies and characterization of the immobilized enzyme layer, Anal. Chem. 52 (1980) 1198-1205.

32. D. Dolman, G. A. Newell, M. D. Thurlow, H. B. Dunford, A kinetic study of the reaction of horseradish peroxidase with hydrogen peroxide, Can. J. Biochem. 53 (1975) 495-501.

33. T. Razgus, L. Gorton, J. Emnéus, and G. Marko-Varga, Kinetic models of horseradish peroxidase action on a graphite electrode, J. Electroanal Chem. 391 (1995) 41-49.

34. M. S. El-Deab and T. Ohsaka, Direct electron transfer of copper-zinc superoxide dismutase (SOD) on crystallographically oriented Au nanoparticles, Electrochem. Commun. 9 (2007) 651-656.

35. H. Liu, Y. Tian, and Z. Deng, Morphology-dependent electrochemistry and electrocatalytical activity of cytochrome $c$, Langmuir 23 (2007) 9487-9494.

36. Q. Rui, K. Komori, Y. Tian, H. Liu, Y. Luo, and Y. Sakai, Electrochemical biosensor for the detection of $\mathrm{H}_{2} \mathrm{O}_{2}$ from living cancer cells based on $\mathrm{ZnO}$ nanosheets, Anal. Chim. Acta 670 (2010) 57-62.

\section{FIGURE CAPTION}

Figure 1. Schematic illustration of a CNTs/G and G electrodes modified with HRP.

Figure 2. (A) CVs of graphene, CNTs/G, HRP/G, and HRP/CNTs/G electrodes in a $0.067 \mathrm{M}$ phosphate buffer solution $\left(\mathrm{pH} 7.4\right.$ ) at scan rate of $100 \mathrm{mV} \mathrm{s}^{-1}$. Relationship between anodic and cathodic peak currents of HRP/G and HRP/CNTs/G electrodes and scan rate.

Figure 3. Dependences of $E_{\mathrm{P}}-E^{0}$ of redox couple for $\mathrm{HRP} / \mathrm{G}$ and $\mathrm{HRP} / \mathrm{CNTs} / \mathrm{G}$ electrodes on the logarithm of scan rate (Laviron plots) in the $0.067 \mathrm{M}$ phosphate buffer solution ( $\mathrm{pH} 7.4)$.

Figure 4. Cathodic current densities of $\mathrm{H}_{2} \mathrm{O}_{2}$ reduction at the graphene, CNTs/G, 
$\mathrm{HRP} / \mathrm{G}$, and HRP/CNTs/G electrodes in the $0.067 \mathrm{M}$ phosphate buffer solution ( $\mathrm{pH}$ 7.4) at $+150 \mathrm{mV}$ vs. $\mathrm{Ag} \mid \mathrm{AgCl}$ 


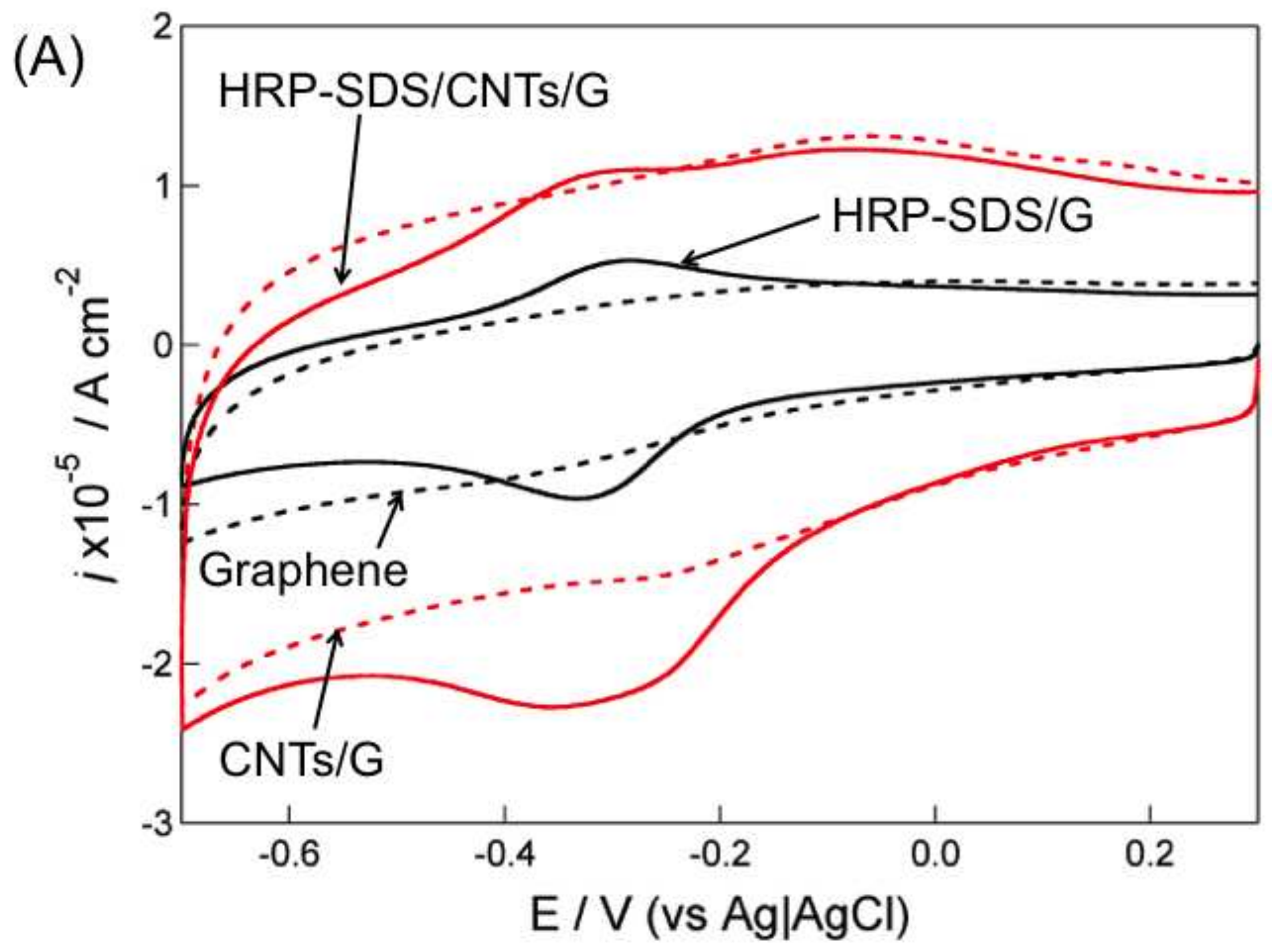




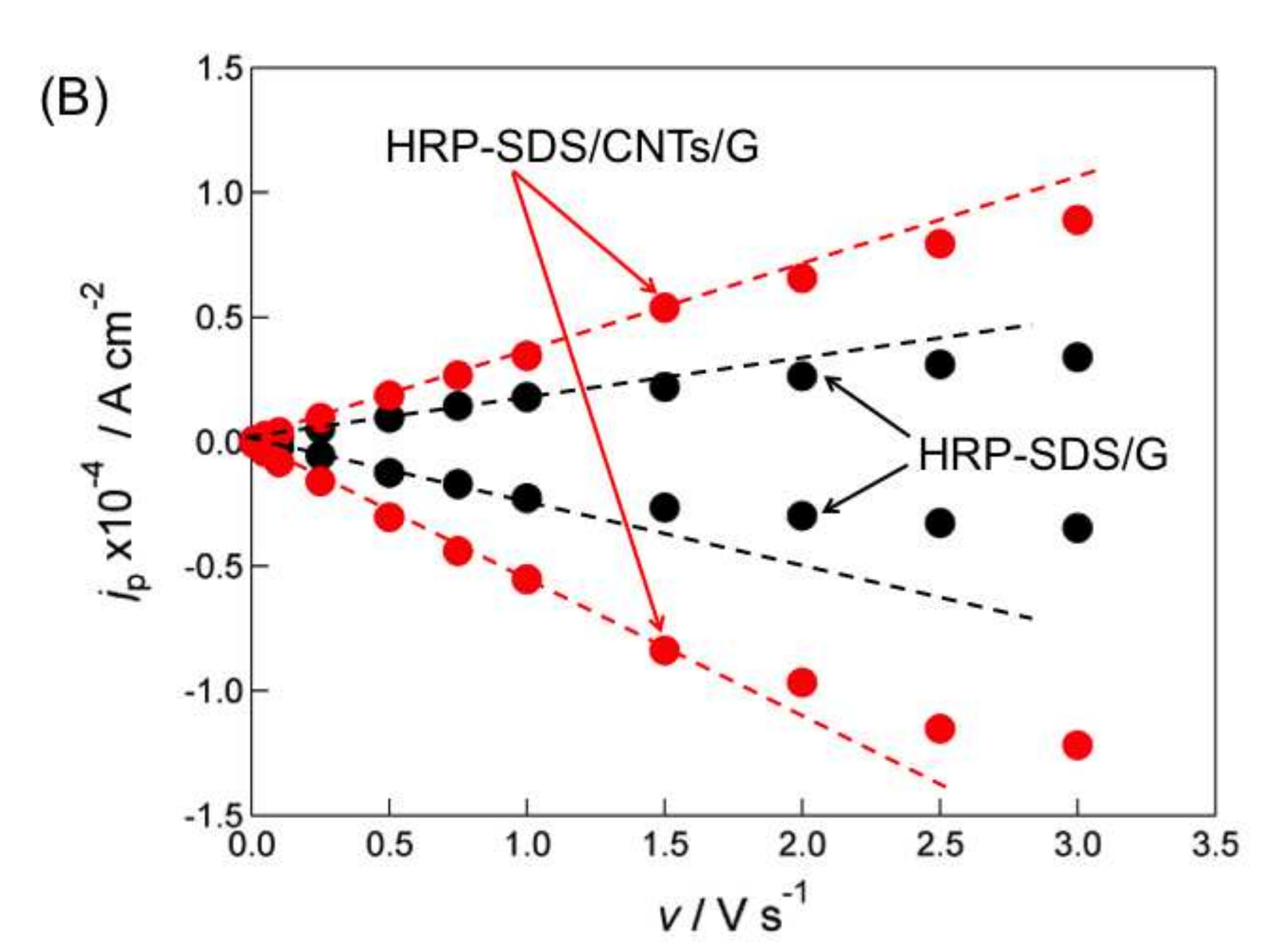




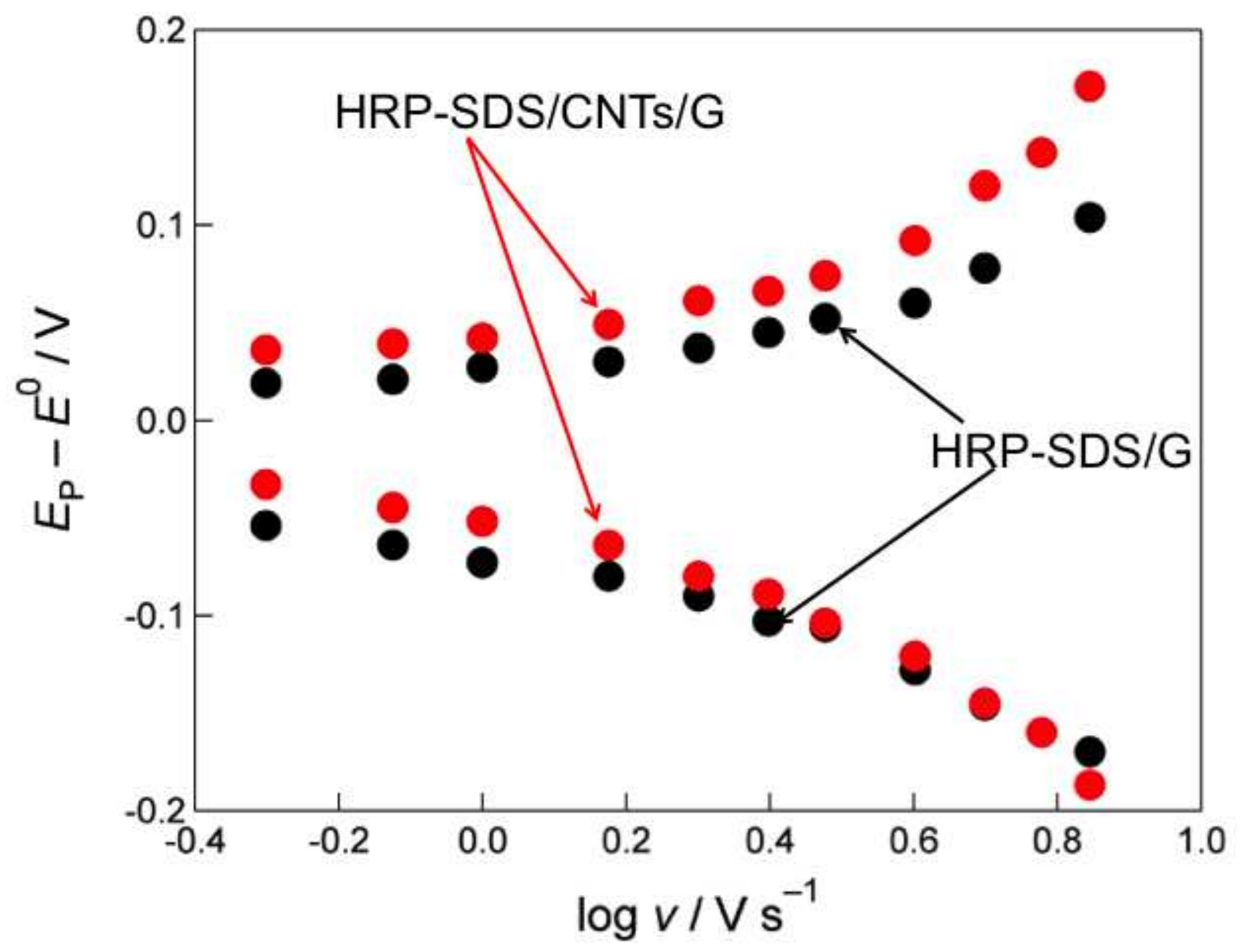




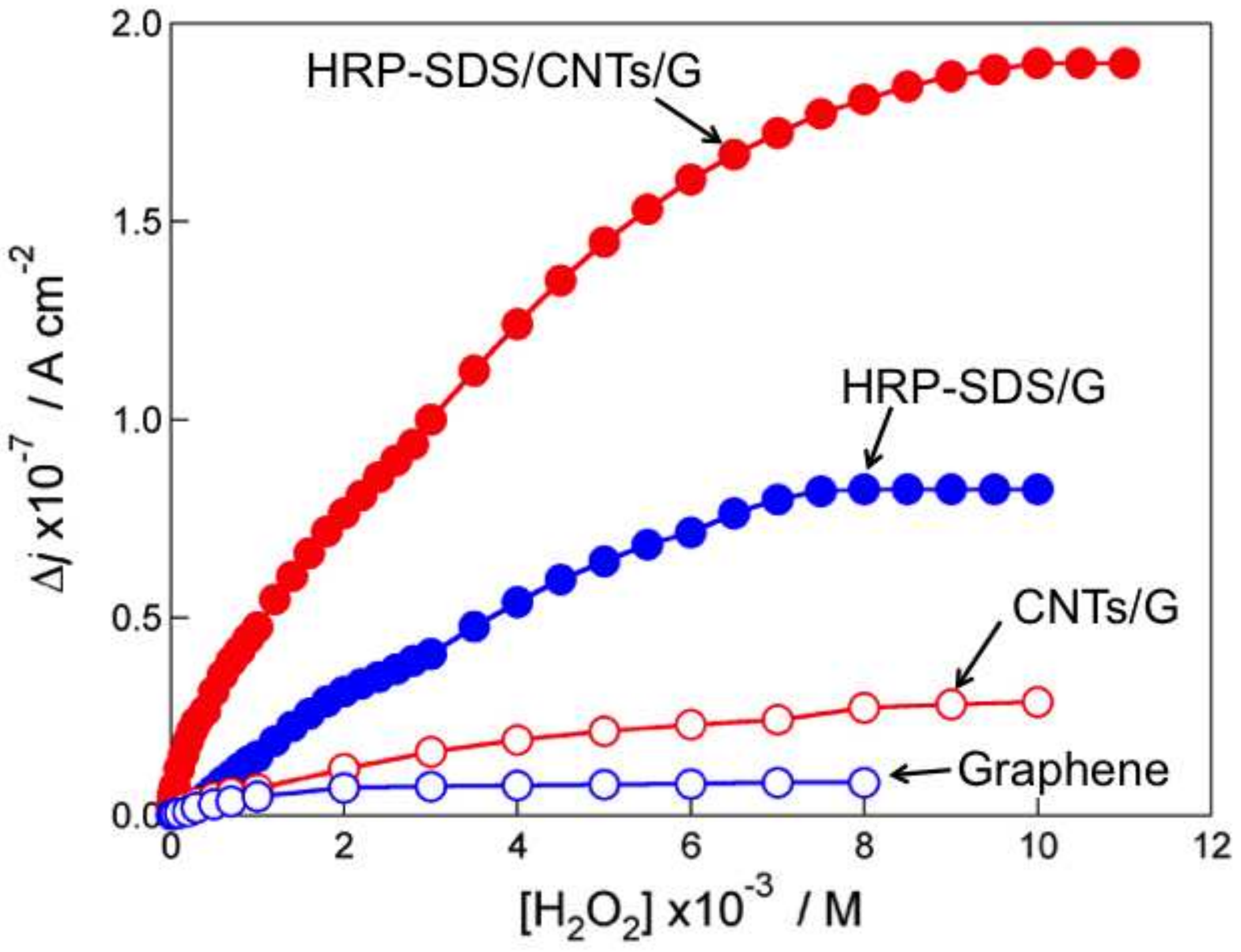




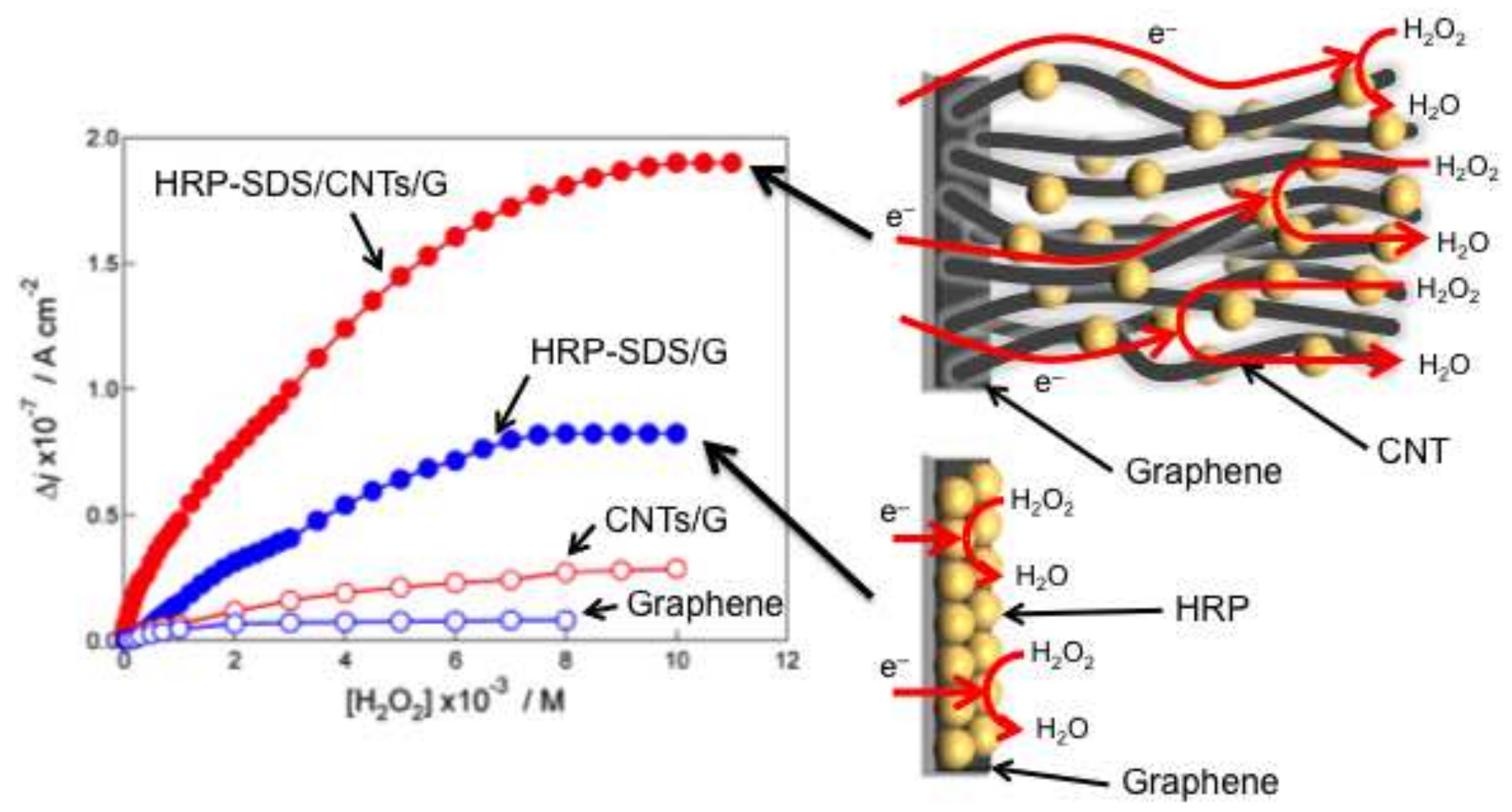

G - graphene; CNT - carbon nanotube; HRP - horseradish peroxidase; SDS - Sodium dodecyl sulfate 